\title{
The Gut microbiota and potential importance in Paediatrics
}

\author{
${ }^{1}$ Sathiadas M G
}

\begin{abstract}
Gut microbiota are commensals seen in the gastrointestinal system having both pathogenic and non-pathogenic effects. Some microbiota that are available commercially have benefits in acute gastroenteritis, necrotising enterocolitis in low birth weight babies and in certain types of allergies. The use of probiotics is debatable in clinical use and it is not recommended in seriously ill immunodeficient children. The duration of the use of probiotics and type of organisms that are commercially available to be used in different disease conditions also have varied recommendations.
\end{abstract}

Key words: Probiotics, Gastroenteritis, Paediatrics

\section{Introduction}

The foetal gastrointestinal tract is sterile but it starts to get colonised with micro-organisms at the time of birth. Both pathogenic and non-pathogenic forms exist together in balance. Probiotics are microorganisms that have health benefits when consumed. According to WHO, the definition of probiotics are microorganisms when administered in adequate amounts confer a health benefit to the host. These microorganisms colonise practically every surface of the human body that is exposed to the external environment, including the skin, oral cavity, respiratory, urogenital and gastrointestinal tract. Of these body sites, the gastrointestinal (GI) tract is by far the most densely colonised organ. The complex community ${ }^{1}$ of microorganisms residing in or passing through the GI tract is referred to as the intestinal microbiota ${ }^{1}$.

The benefits were first described by the Nobel Prize winner Eli Metchnikoffin in 1907 when he described the dependence of the intestinal microbes on the food, makes it possible to modify the flora in our bodies and to replace the harmful microbes by useful microbes. Then the WHO/FAO described the guidelines for evaluation of probiotics in food in 2001. In October 2013, a group of scientific experts assembled in London, UK to discuss the scope and appropriate use of the term 'probiotic' and the conclusions were published in June $2014^{2}$.

\section{Microbial diversity}

The intestinal microbiota plays a role in metabolic, nutritional, physiological and immunological processes in the human body. There are numerous

\footnotetext{
${ }^{1}$ Senior lecturer in Paediatrics, Faculty of

Medicine, University of Jaffna.
}

organisms and each having several species and subspecies imparting different functions. Some of these micro-organisms are resident and some are transient residents in relation to the food consumed. Over 400 species have been identified to be colonised in the gut ${ }^{3}$.

Seven bacterial divisions constitute the gut microbiota i.e. Firmicutes, Bacteroides, Proteobacteria, Fusobacteria, Verrucomicrobia, Cyanobacteria, and Actinobacteria, with Firmicutes and Bacteroides being the most abundant species. Bacterial communities exhibit quantitative and qualitative variations along the length of the gastrointestinal tract due to various host factors like $\mathrm{pH}$, transit time, bile acids, digestive enzymes, and mucous and non-host factors like nutrients, medication, and environmental factors. The bacterial factors such as enzymes adhesion capacity and metabolic activity also contribute to the presence of microbiota in the gut 4 .

\section{Functions of Probiotics}

\section{Ensuring protection}

Pathogen displacement and protection of the gut from pathogenic organisms is an accepted function of the gut microbiota. Commensal organisms prevent pathogenic colonization by competing for attachment sites and nutrients, and also through the production and secretion of antimicrobials. Those mechanisms are relevant for reducing the level of lipopolysaccharides, peptideglycans, bacterial CpG-DNA motifs, and superantigens, which can all be detrimental to the host. The indigenous microbiota is also essential for development of the immune system.

The microbiota plays a major role in intestinal structure and function. The mucus layer, which reflects the balance between mucus secretion and bacterial degradation, constitutes an obstacle to the uptake of antigens and pro-inflammatory molecules. There is evidence indicating that butyrate released from the microbiota reinforces the colonic defense barrier by inducing the secretion of mucins.

Some bacterial communities may strengthen the barrier at the level of the tight junctions. Activation of the innate and adaptive immunity along with the regulation of inflammation helps in the protective functions of the gut ${ }^{3}$. 


\section{Metabolic functions}

Production of vitamin K and B group is the main nutritional function. In addition amino acid biosynthesis, bile acid biotransformation fermentation of non-digestible substrates and mucous are some of the functions. Production of short chain fatty acids is also seen which acts as an energy source ${ }^{2}$.

\section{Structural and histological functions}

The gut microbiota promotes epithelial cell growth and differentiation, intestinal villi and crypt development as well as micro-vascularization. One major function is barrier fortifications by promoting tight junctional permeability and mucous layer production ${ }^{2}$. The functions mentioned above overlap with each other (figure1). For example, antimicrobial secretion is a metabolic and protective function of the gut microbiome.

\section{Gut Dysbiosis}

Dysbiosis is a state in which the microbiota becomes altered as a consequence of an alteration in the composition of the microbiota, a change in bacterial metabolic activity, and /or a shift in local distribution of communities. Many factors can alter the gastrointestinal ecosystem, including antibiotics, psychological and physical stresses, radiation, altered peristalsis, and dietary changes. Figure 2 shows various mechanisms involved in gut dysbiosis and its effects.

\section{Altered state of gut microbiome and paediatric disease}

\section{a.Allergies and immune mediated diseases}

The increasing frequency and incidence of atopic diseases such as eczema, asthma, and food allergies are linked to alterations of the intestinal microbiota. Delays or changes in the core microbiome could also potentially affect the development of the immune response. Epidemiologic data provide further evidence that infants delivered via cesarean delivery have higher incidences of atopic diseases such as asthma and type 1 diabetes and food allergies compared with vaginally delivered infants. The possible explanation is due to differing gut mirobes ${ }^{7}$.

Inflammatory bowel diseases like Crohn's disease and ulcerative colitis are linked to change in the microbial organisms. There is evidence that species belonging to the normal gut microbiota are involved in the etiology and/or maintenance of inflammatory processes. Reduced microbial diversity, increased Bacteroidetes and Enterobacteriaceae, and decreased Firmicutes were all observed in patients with inflammatory bowel diseases ${ }^{4}$.

Some allergic conditions are also said to be triggered by alteration in the gut microbiome. A study reported higher levels of Bifidobacteriumadolescentis and Lactobacilli Group I (Lactobacillus rhamnosus, L. paracasei, L. casei) in fecal samples of nonallergic compared with allergic children ${ }^{8}$.

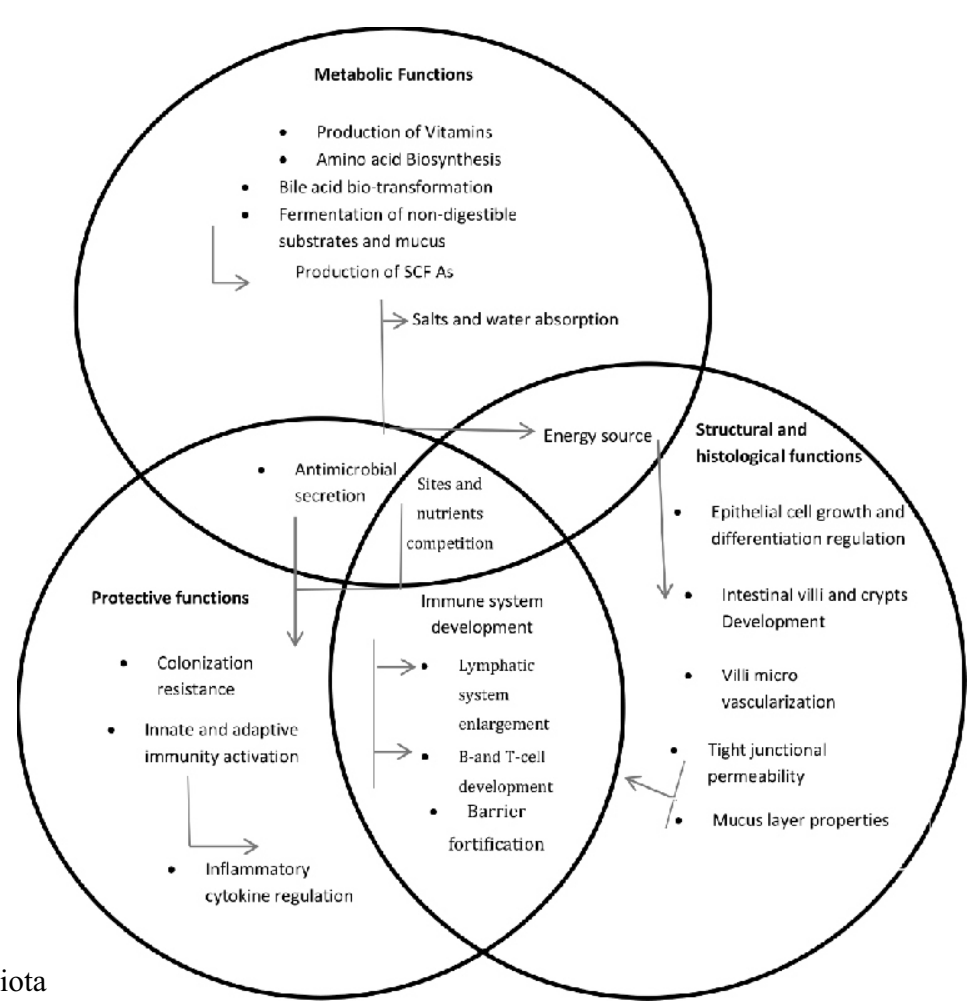






Figure 2: Mechanisms involved in dysbiosis

\section{b.Metabolic disease}

Obesity is one major factor associated with altered gut microbes. Several studies have shown a causal effect of microbial dysbiosis on the development of obesity. A study was initiated which demonstrated that total short-chain fatty acids were higher in an obese group and individual short-chain fatty acid proportions shifted towards propionate in overweight subjects. The ratio of Firmicutes to Bacteroidetes changed in favor of the Bacteroidetes in overweight $(\mathrm{P}=0.001)$ and obese subjects $(\mathrm{P}=0.005)$. Short-chain fatty acid metabolism might play a considerablerole in obesity".

\section{c.Bacterial infections}

Pathogenic bacteria can invade the gastrointestinal tract and infect the body, producing sepsis, shock, multisystem organ failure, and death of the host. The mechanisms by which pathogens overcome obstacles to achieve successful infections are uncertain. Pathogenic infections might be facilitated by disruption of the intestinal ecosystem by environmental factors. An overgrowth of Enterobacteriaceae was observed and the inflammatory response, triggered by the invading pathogen, may function to enhance its colonization, further facilitating its virulence. Thus, alteration of the gut microbiota, initiated by host and environmental factors, may participate in the initiation of diseases caused by infectious agent.

\section{Clinical uses of Probiotics in Paediatrics}

\section{Prevention and treatment of infectious diarrhoea}

Several studies have shown the benefit of these probiotics in the use of prevention and treatment of infective diarrhoea. In regards to prevention of infective diarrhoea, in a double-blind, placebocontrolled trial by Weizman et al, 201 infants (4-10 months of age) received either a probioticsupplemented formula containing either $B$ lactis or $L$ reuterior a control formula without an added probiotic over a 12-week study period. The study was conducted at 14 different child care centres over a 2year period. Infants who were fed a probiotic supplemented formula had fewer and shorter episodes of diarrhoea than did infants in the control group $^{10}$. The available data do not support routine use of probiotics to prevent rotavirus diarrhoea. However, there may be special circumstances in which probiotic use in children in long-term health care facilities or in child care centres is beneficial to prevent the diarrhoea ${ }^{6}$.

Probiotics administered early in the treatment of diarrhoeal disease has shown benefit. Randomized controlled trials in healthy children in developed countries have provided data on the therapeutic benefit of probiotics in children with acute infectious diarrhoea. In a randomized, doubleblind, placebo-controlled trial by Szymanski et al, administration of Lactobacillus rhamnosus GG (LGG)significantly shortened the duration of acute rotavirus diarrhoea by a mean of 40 hours, but duration of diarrhoea of any other aetiology was not affected. Probiotic administration also shortened the time necessary for intravenous rehydration by a mean of 18 hours. LGG is the most effective probiotic reported to date ${ }^{6}$.

\section{Prevention and treatment of antibiotic induced diarrhoea}

Another important use of probiotics is in prevention of antibiotic induced diarrhoea. Treatment with 
probiotics compared with placebo has reduced the risk of developing antibiotic-associated diarrhoea from $28.5 \%$ to $11.9 \%$ (relative risk [RR]: 0.44 [95\% CI: $0.25-77] ; \quad P<0.006)$. LGG, Blactis, $S$ thermophilus, and $S$ boulardii have been the most common agents used in RCTs. Approximately 1 in 7 cases of antibiotic associated-diarrhoea was prevented by the use of a probiotic ${ }^{11}$.

\section{Prevention and treatment of atopic diseases}

The risk of allergic disease explained by the hygiene hypothesis could well be the result of a lack or delay in the eventual shift of the predominant $\mathrm{T}$ helper 2 type of response to more of a balance between Th1- and Th2-type responses. A Cochrane review summarised in 2008 showed probiotics have not yet been proven to be effective in the treatment of eczema ${ }^{12}$.

\section{Prevention of Necrotising Enterocolitis (NEC) in low birth weight babies}

The pathophysiology of NEC appears to be multi factorial, with premature birth being the most pronounced risk factor. Other factors in the development of NEC include intestinal immaturity, an excessive intestinal inflammatory response to microbial stimuli, colonization by diseasepredisposing microbial populations in the GI tract and prolonged use of antimicrobials. In a 2008 Cochrane review based on 9 RCTs, enteral probiotic supplementation significantly reduced both the incidence of NEC (stage II or more) (RR 0.32 [95\% CI: 0.17-0.60]) and mortality (RR: 0.43 [95\% CI: $0.25-0.75]$ ). A total of 1425 infants who were born at less than 37 weeks' gestational age and/or less than $2500 \mathrm{~g}$ birth weight were included in this meta-analysis. No systemic infections or serious adverse events that were directly attributed to the administered probiotic organism were reported for these RCTs. All these studies have used a mixture of probiotics namely LGG, Bifidobacterium breve, Saccharomyces species, and mixtures of Bacteroidesbifidus, $S$ thermophilus, Lactobacillus acidophilus, and Bifidobacterium infantis, which shown a proven benefit ${ }^{12}$.

\section{Other disorders}

Beneficial effects have been shown in the treatment of ulcerative colitis, Crohns disease and irritable bowel disease. Several studies have been done, but none shows a proven benefit. As yet, no published RCTs recommend the routine administration of probiotics to either treat or prevent cancer in adults or children ${ }^{4}$.

\section{Safety of probiotics}

To date the probiotics are safe in otherwise healthy children. Few cases have been reported in literature where probiotics use has led to sepsis. Sepsis has been reported in severely ill and chronically ill with immunodeficiency. The Committee on Nutrition of the European Society of Pediatric Gastroenterology, Hepatology and Nutrition concluded that more studies are required to establish the safety and efficacy of probiotic and prebiotic products in children. Centralized oversight and probiotic product monitoring was also recommended in a report from the Food and Agriculture Organization of the United Nations World Health Organization ${ }^{2}$.

\section{Conclusion}

Several beneficial effects have been observed especially in the treatment of diarrhoea with probiotics especially in healthy children; but, important questions remain unclear establishing the clinical applications for probiotics, including the optimal duration of probiotic administration as well as preferred microbial dose and species. The longterm impact on the gut microflora in children is also unknown despite having short term benefits.

\footnotetext{
Summary

- Probiotics are micro-organisms that have health benefit

- Breast milk is a good source of probiotics in the first 6 months of life

- Probiotics are beneficial in the treatment and prevention of diarrheal diseases in healthy normal infants and children

- Evidence suggests probiotics reduce the incidence of NEC in low birth weight babies

- Some beneficial effect is reported in atopic and allergic conditions

- Beneficial effects in inflammatory bowel disease, irritable bowel disease and malignancies are not established

- Probiotics should be avoided in seriously or chronically ill children

- Optimal duration, the preferred microbial species and dose remains unclear
} 


\section{References}

1. Ardythe L Morrow, Anne J Lagomarcino, Kurt R Schibler, Diana H Taft1, Zhuoteng Yu, Bo Wang; Early microbial and metabolomic signatures predict later onset of necrotizing enterocolitis in preterm infants; Microbiome 2013, 1:13

2. Colin Hilletal, EXPERTCONSENSU $S$ DOCUMENT The International Scientific Association for Probiotics and Prebiotics consensus statement on the scope and appropriate use of the term probiotic, Nature reviews gastroenterology and hepatology, AUGUST 2014 | VOLUME 11

3. Dan W. Thomas, Frank R. Greer and Committee on Nutrition;Probiotics and Prebiotics in Pediatrics, Pediatrics2010;126;1217

4. Coreen L. Johnson and James Versalovic; The Human Microbiome and Its Potential Importance to Pediatrics; PEDIATRICS Volume 129, Number 5, May 2012, p1-11

5. Lorenzo Morelli, Postnatal Development of IntestinalMicroflora as Influenced by Infant Nutrition; J. Nutr. September 2008 vol. 138 no. 9 1791S-1795S

6. Szymanski H, Pejcz J, Jawien' M, Chmielarczyk A, Strus M, Heczko PB. Treatment of acute infectious diarrhoea in infants and children with a mixture of three Lactobacillusrhamnosus strains: a randomized, double-blind, placebo-controlled trial. AlimentPharmacolTher. 2006;23(2):247-25)

7. Negele K, Heinrich J, Borte M, et al; LISA StudyGroup. Mode of delivery and development of atopicdisease during the first 2 years of life. Pediatr Allergy Immunol. 2004;15(1):48-54)

8. Sjogren YM, Jenmalm MC, Bottcher MF, BjorkstenB, Sverremark- Ekstrom E. Altered early infant gut microbiota in children developing allergy up to 5years of age. ClinExp Allergy. 2009;39:518-526

9. Schwiertz A, Taras D, Schafer K, et al. Microbiota andshort-chain fatty acid in lean and overweight healthysubjects. Obesity (Silver Spring). 2010;18:190-195

10. Weizman Z, Asli G, Alsheikh A. Effect of a probiotic infant formula on infections inchild care centers:comparison of two probiotic agents. Pediatrics.2005;115(1):5-9)

11. Szajewska H, Ruszczynski M, Radzikowski A. Probiotics in the prevention of antibioticassociated diarrhea in children: a meta-analysis of randomized controlled trials. $J$ Pediatr. 2006;149(3):367-372

12. Alfaleh K, Bassler D. Probiotics for prevention of necrotizing enterocolitis in preterm infants. Cochrane Database Syst Rev. 2008;(1):CD005496 